\title{
The investigation of TRB auto-control cutting system
}

\author{
Wu Zhiqiang ${ }^{1, a}$, Wang Jun ${ }^{1, b}$, Sun Tao ${ }^{1, c}$ \\ ${ }^{1}$ The State Key Laboratory of rolling and automation, Northeastern University, Shenyang, 110819, \\ China

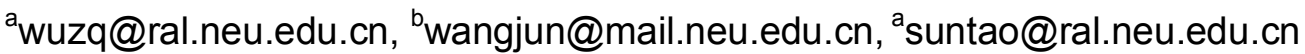

Keywords: TRB, cutting system, shape Matching

Abstract: The efficiency of shear line is the bottlenecks of TRB production and quality, the developed TRB automatic cutting system by re-identification of the shape parameters of incoming period variable thick strip, according to the requirements of the finished board, cutting optimization program completed strip automatic positioning, to achieve accurate cutting purposes.

\section{Introduction}

Car weight is a key factor to reduce vehicle exhaust pollution. The use of variable thickness board (TRB) to reach the car weight loss purposes have been widely shape, position, size, to the automotive industry recognition. After rolling cycle variable thickness plates subject to annealing, then straightening, final length cross cutting get TRB board. The TRB boards through stamping or through subsequent processing steps, eventually become auto parts

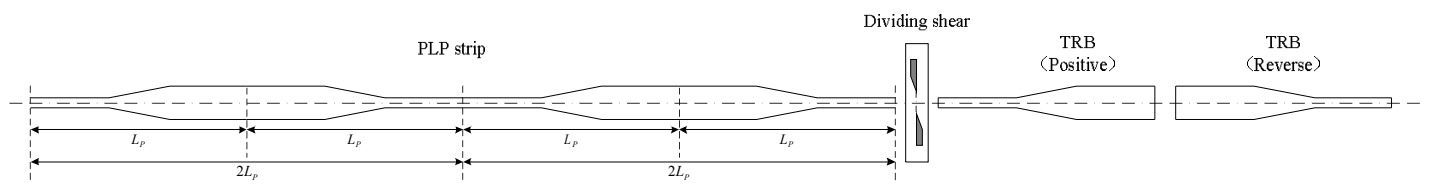

Fig.1. TRB board production process

Before variable thickness plate entering the shearing process, the plate should go through cycles of rolling, annealing and straightening processes, due to the rolling process periodically rolling roll gap adjustment range, the expansion and contraction of the strip during the annealing process, and straightening process of the micro-extension, these factors will make the strip slight changes in the longitudinal direction, so that the cycle length of the strip changes. For this change, it is irrelevant that the constant thickness cut to length, but it cannot be ignored to the variable thickness plate. It is not only influence the appearance of a single block TRB plate, but also influence multiple errors in a row after a cumulative TRB board cut. The cutting position has to be re-adjusted. Thus it badly reduces production efficiency.

Before cutting cycle of incoming strip, the control system analysis the shape of TRB board, so that precise positioning shears TRB Strip. At the same time capable of continuous shear cumulative error arising after judgment, optimized cut.

\section{The shear line equipment of TRB board}

The automatically cut line of TRB board is shown in Figure 2, including uncoiler, pinch roll before straightening, straightening machines, shearing buffer strip pits, pinch roll, thickness, pinch before cutting rolls, shears and delivery platforms and other equipment. The uncoiler's speed pulse encoder settings for unwinding strip line speed, tension control; the leveler in the high-speed pulse encoder is used to control the unwinding of the strip to match the line speed; before straightening 
pinch rollers, front clip gage rolls, pinch roller speed pulse encoder configuration before cutting, shearing buffer for complete control, cut strip belt position tracking; thickness and pinch roller encoder strip PLP together to complete each cycle dimensions identification.

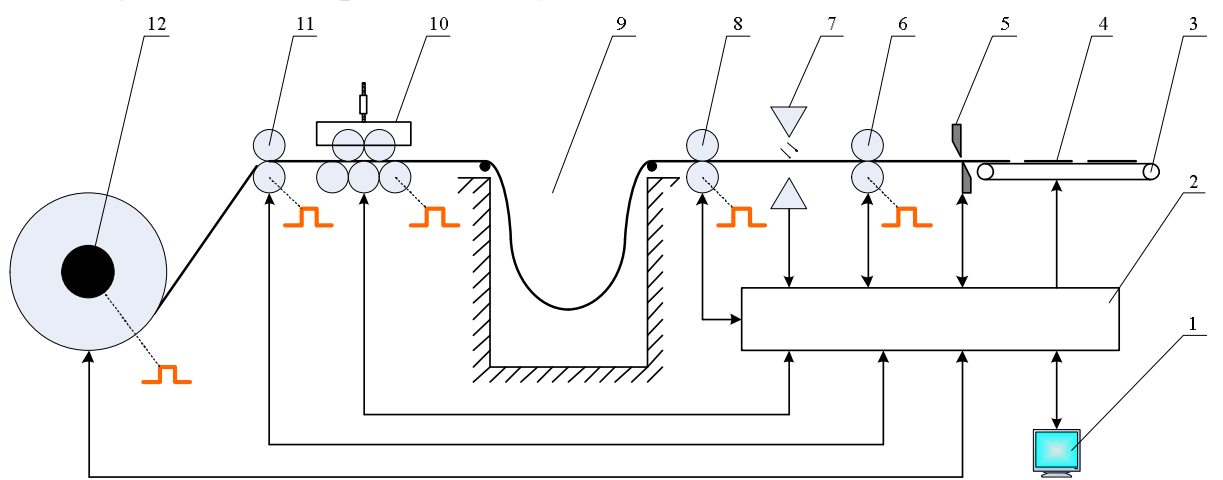

1- HMI computers, 2 computer controlling system, 3 delivery platform planks poor shear completed

4-,TRB 5- shears, 6- scissors before pinch rolls, 7-gauge, measuring 8- thick instrument before pinch rolls, 9- hole cut buffer strip, 10-leveler, 11-pinch rolls before straightening, 12-uncoiler

Fig.2. TRB plate shear line equipment chart

\section{The automatically controlling process of cutting TRB board}

The automatically controlling process of cutting TRB board is shown in figure 3 .

First, diverse thick slab's these parameters include dimensions parameters, PLP incoming strip coil diameter, shear rate and buffer pit buffer strip length to determine the initial shear parameters of diverse thick slab.

Then, PLP with volume uncoiler uncoiling, followed through with straightening before pinch rolls, leveler, pinch roll front thickness, thickness, pinch rolls before scissors, shears, each followed by pressing the pinch roller tight strip. Established micro tension by unwinding machine, so tight straight strip, hydraulic shears cut the lead.

Third, after starting the automatic cutting control, PLC controlling system starts unwinding length PLP strip tracking, automatic controlling buffer strip pit length; when the length of the PLP strip is less than the set buffer length of the strip, automatic uncoiler feeding.

Fourth, PLC controlling system will automatically recognize the PLP strip's dimensions, precise tracking the position of TRB board, complete cutting position control; during the process of sending plates, PLC controlling system can determine optimization cut programs and eliminate the accumulated error, by comparing the dimensions of the current cutting TRB board.

Fifth, TRB is transported to the downstream process including inspection, sorting, packing by conveyor.

\section{Controlling System Components}

The universal, open, high speed, high reliability, ease of upgrade and expansion of the principles of the TRB board controlling system and automatic cutting system accommodate the need to control technology continues to develop and reserve development. Due to the algorithm of the target identification of PLP strip and half-cycle plate is the core of the entire PLP strip automatic cutting control system. The Shape matching algorithm involves shape feature extraction and similarity measure describing technology, algorithm complexity. The automatic cutting system of PLP strip is a real-time control system based on PLC. In summary the identification and matching algorithms of TRB board must be efficient and accurate. 


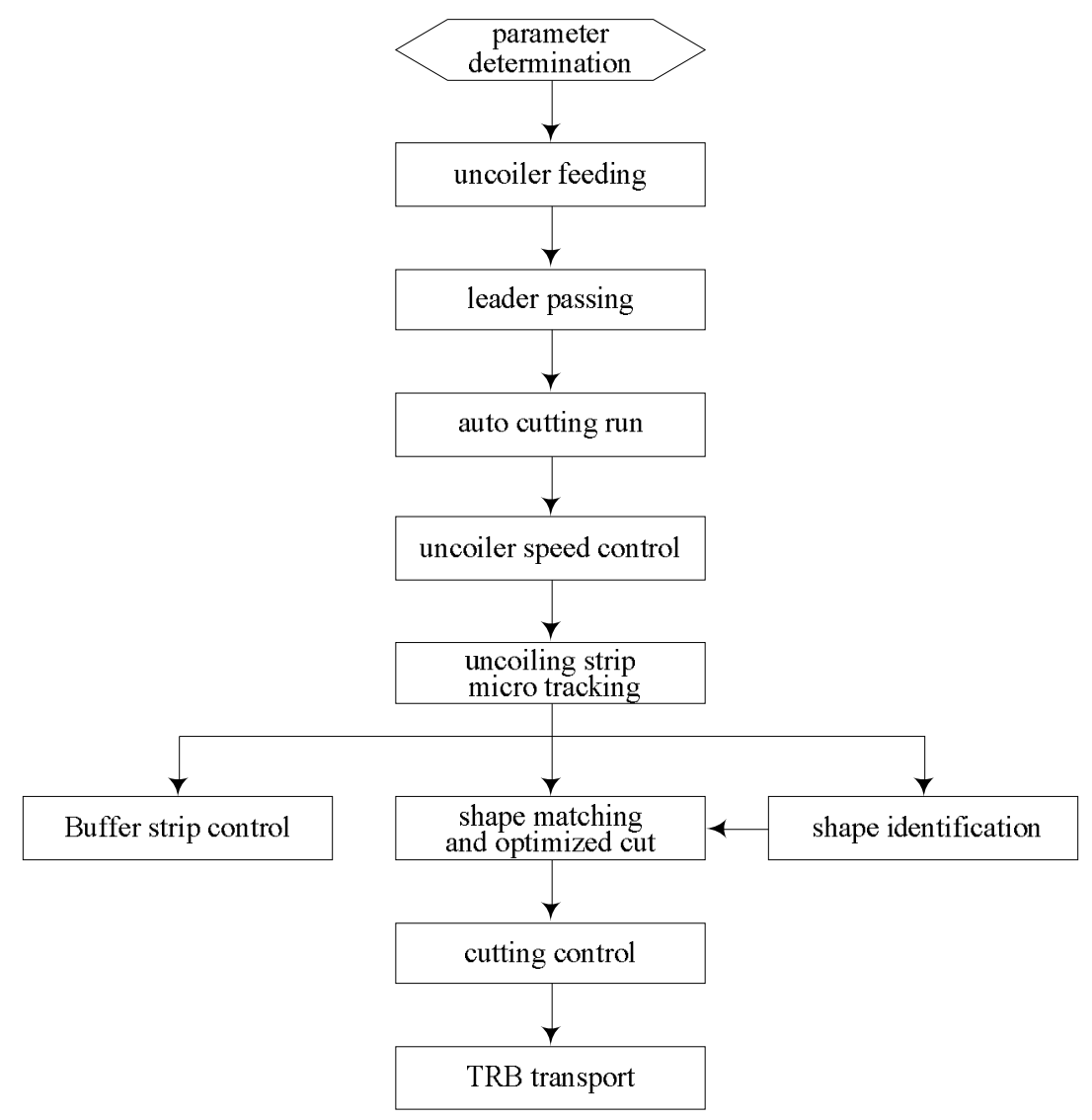

Fig.3. TRB plate shearing process

TRB plate shear control system is shown in Figure 4. SIEMENS S7-400 series PLC is the core of basic automation level control system which complete the cut line sequence control and a variety of closed-loop control tasks, while collecting shear line thickness, encoder data and upload to the co-processing computer. The main computer complete TRB plate shape identification and matching cut strategy development and optimization. Using co-processing can improve the accuracy and speed of TRB board computer matching.

Siemens Wincc is used in HMI which is used to implement and monitor of the entering original data of cutting process. Event management system can be used to obtain HMI automation system events, such as buffering, storage, display and analysis, including time recording. Mass storage measurement and control data to provide data, charts, graphs and other means to achieve diagnostic monitoring and analysis capabilities.

Transmission equipment, instrumentation and other equipment constitute the Device-level of controlling system. In uncoiler and straightener of the system, the variable frequency drive is used as speed regulator, complete unwinding and straightening of control tasks; in order to achieve better precision strip length transfer, transmission before cutting threw roll servo-control system. 


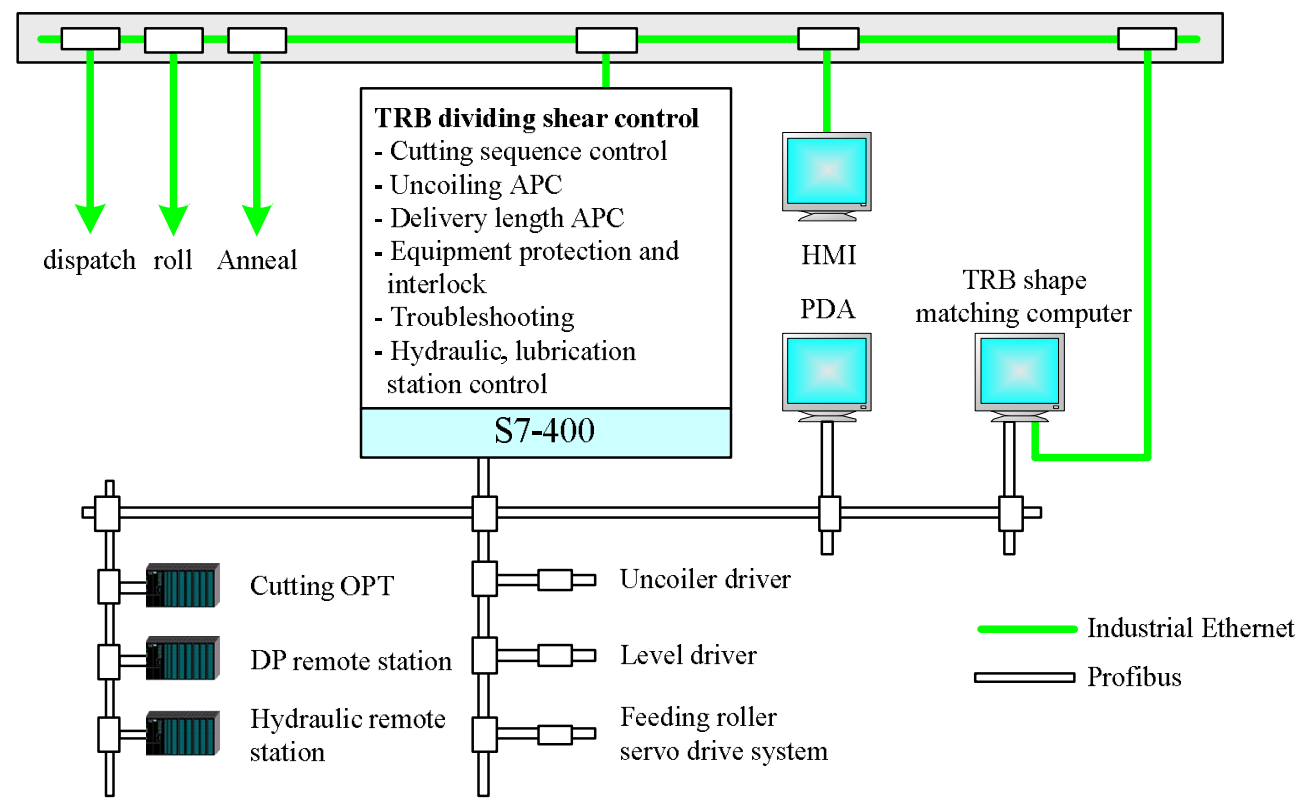

Fig.4. TRB board cut automatic control system structure diagram

PDA computer while the system configuration, is the use of DP bus communication mode, the sampling period of up to $1 \mathrm{~ms}$, by analyzing the collected data to quickly diagnose problems, eliminate potential failure factors, improve production efficiency.

The data transfer between TRB shear automatic control system and control room, rolling zone, annealing zone through central switch, form a unified coordination and management of production data.

\section{Key algorithms of controlling process}

The algorithm of the target identification of PLP strip and half-cycle plate is the core of the entire PLP strip automatic cutting control system. The Shape matching algorithm involves shape feature extraction and similarity measure describing technology, algorithm complexity. The automatic cutting system of PLP strip is a real-time control system based on PLC. In summary the identification and matching algorithms of TRB board must be efficient and accurate.

\section{PLP strip shape identification algorithm}

Thickness measurement point set distance to the hydraulic shears to cut the line. In thickness measurement of the $\mathrm{x}$-axis coordinate zero point, to measure the thickness of the $\mathrm{y}$-axis, establishing PLP strip Dimensions coordinate system. Set with N coordinates data stack, used to record the length of the strip dimensions.

$$
A\left\{\left(n, h_{n}\right),\left(2 n, h_{2 n}\right),\left(3 n, h_{3 n}\right), \cdots,\left(N n, h_{N n}\right)\right\}
$$

Where: $\mathrm{n}$ - for identification accuracy, due to the requirements of the precision of finished various thick slab $(\leqslant \pm 1 \mathrm{~mm}), \quad n=0.1 \mathrm{~mm}$;

$$
N \text { - The number of array, } N=\left\lceil L_{0} / n\right\rceil,\lceil\rceil \text { Said the roundup. }
$$

Based on first-in first-out principle, whenever the accumulated value of front pinch roller conveyor belt gage length equal to an identification accuracy $n$, forthcoming in the identification precision sampling strip thickness averages hn into the stack, and then start the next one sample identification accuracy.

Because PLP strip belt slippage in the process, considering the thickness of the pinch roller and cut before the pinch roller conveyor belt length, in order to improve the tracking accuracy. 
Therefore, the identification accuracy strip belt tracking $L_{n}$ :

$$
\sum_{n}=\sum_{i=1}^{m}\left(L_{H}(i)+L_{C}(i)\right) / 2
$$

\section{TRB shape matching algorithm}

Set goals difference slab length, length and thickness of the slab goal difference curve:

$$
h=f(l)
$$

Where: $l \in\left[0, L_{P}\right]$ 。

Because PLP strip a period ending by two planks connected difference constituted by the length cut after cut TRB board is positive and negative phases. Therefore, setting two coordinate stack has $M$ coordinates, within each stack being used to record the anti-two arrangement TRB board shape curve:

$$
\begin{aligned}
& B\{(n, f(n)),(2 n, f(2 n)),(3 n, f(3 n)), \cdots,(M n, f(M n))\} \\
& C\{(n, f(M n)),(2 n, f((M-1) n)),(3 n, f((M-2) n)), \cdots,(M n, f(n))\}
\end{aligned}
$$

Where: $\mathrm{n}$ - for identification accuracy, due to the requirements of the precision of finished various thick slab $(\leqslant \pm 0.5 \mathrm{~mm}), \quad n=0.1 \mathrm{~mm}$;

$$
N \text { - The number of array, } N=\left\lceil L_{0} / n\right\rceil,\lceil\rceil \text { Said the roundup }
$$

The ordinate coordinates of last $\mathrm{M}$ of A stack compare with corresponding coordinate of B stack, respectively, the following formula:

$$
D\left\{\left|f(n)-h_{(N-M) n}\right|,\left|f(2 n)-h_{(N-M+1) n}\right|,\left|f(3 n)-h_{(N-M+2) n}\right|, \cdots,\left|f(M n)-h_{N n}\right|\right\}
$$

When the elements of D stack were less than $\delta$, a TRB board which matches the size distribution requirements of the $\mathrm{B}$ stack has reached the shear line, general admission. $\delta=0.01 \mathrm{~mm}$

Same reason for stack $E$ :

$$
E\left\{\left|f(M n)-h_{(N-M) n}\right|,\left|f((M-1) n)-h_{(N-M+1) n}\right|,\left|f((M-2) n)-h_{(N-M+2) n}\right|, \cdots,\left|f(n)-h_{N n}\right|\right\}
$$

When the elements of E stack were less than $\delta$, a TRB board which matches the size distribution requirements of the $\mathrm{C}$ stack has reached the shear line, general admission. $\delta=0.01 \mathrm{~mm}$

When the TRB board of a B or C stack size arrive the shear line, the strip was cut the front waste out, and then cut back waste out, ultimately qualified TRB board.

\section{Conclusion}

Length shear cutting precision is the key production processes of TRB board product. The cutting efficiency is constrained capacity bottlenecks. According to the characteristics of TRB board, designed length cut production line and automatic control system passed the production commissioning. Now the system is working properly. Cutting length precision automatic cutting system $<1 \mathrm{~mm}$, shape matching a pass rate of $99 \%$, rate of finished is $98 \%$. The system not only meets production requirements, but also has the potential to further increase capacity. 


\section{Acknowledgments}

The authors would like to acknowledge the support of the Fundamental Research Funds for the Central Universities (N120307001, N120407005).

\section{References}

[1] Zhang Guomin, Yin Jianping, Zhu En, and Mao Ling. 2D Shape Matching Based on Polar Interval Operations[J], JournaI 01 Computer Research and Development,2008(45S).286-290

[2] Belongie S, Malik J, Puzicha J. Shape matching and object recognition using shape contexts[J]. IEEE Transactions On Patten Analysis And MachineIntelligence. 2002, 24(4): 509-522.

[3] HU Yan-ping* , MA De-cheng, HE Hong-peng, OU Zong-ying. Principle and method of graphic matching based on model reconstruction[J]. Journal of Dalian University of Technology. 2005,45(2):221-225

[4] Peng Shaowu, Lin Liang, Liu Leyuan, Sang Nong. Object matching using shape features[J]. J.Huazho ng Univ .of Sci.\& Tech .(Natural Science Edition), 2009,37(3):58-61

[5] H.wang, M.Brady. Real-time corner detection algorithm for motion estimation[J].Image and Vision Computing. 1995,13(9):695-70

[6] GuoWenHui. PLC in the industrial production of advantages in shallow [J], heilongjiang science and technology information, 2004 (10). 69-72

[7] Du Jitao, Gan Yi, Qi Congqian. Tailor Rolled Blanks and Keys Technologies in Its Rolling Applications[J]. Automobile Technology, 2007(7): 45-48.

[8] Shi Zhigang, Wang Hongyan. Application of the Tailor Rolling Blanks in the Body Lightweight[J]. Shanghai Auto, 2008(8): 36-39.

[9] Zhang Hongmei, Yu hailong. Tailor-made Metal Sheet and Its Application in Automotive Industry[J]. Automobile Technology \& Material, 2003(03):20-22.

[10] Qi Congqian. The application of Tailor Rolled Blanks in Automobile. Automobile \& Parts Technology[J], 2006,47:38-39.

[11] Cui Huaiyang, Zuo Tiechuan. Tailored laser-welded blanks and its application in automobile manufacturing[J]. Welding Technology, 2004(01),Vol 33:23-27. 